Jonas Tiepmar, Jens Mittelbach, Melanie Kaiser, Daniela Dobeleit, Paul Schwanse, Uta Fröhner und Maik Jähne

\title{
Wissen kommt von Machen
}

\section{Zukunftsgestaltung in wissenschaftlichen Bibliotheken im Zeitalter der Digitalisierung}

https://doi.org/10.1515/bfp-2018-0009

Zusammenfassung: Mit der Digitalisierung und der damit einhergehenden technologischen Revolution verschwinden die Grenzen der früher deutlich getrennten internen und externen Anteile der publizistischen Produktionskette. Außerdem befördert das Aufkommen von neuartigen und leicht zugänglichen Technologien wie 3D-Druck oder Lasercutting den Methodenwandel in der Wissenschaft und kommt interdisziplinären Ansätzen entgegen. Der Bedarf an Wissen zu diesen Technologien und dessen Vermittlung steigt aber zeitgleich mit. Die SLUB Dresden versucht, diesem Bedarf mit dem Angebot eines wissenschaftlichen Makerspace zu entsprechen. Erfolge dieses Konzeptes und Herausforderungen bei der Umsetzung werden hier beschrieben.

Schlüsselwörter: Makerspace; Fablab; Digitalisierung; wissenschaftliche Bibliothek; Zukunftskonzept; Strategieplanung

\section{Knowledge Comes from Doing \\ Shaping the Future in Scientific Libraries in the Age of Digitalization}

Abstract: The technological revolution in the age of digitalization brings blurs the boundaries of the formerly distinct internal and external parts of the publishing production chain. In addition, the emergence of new and easily accessible technologies such as 3D printing or laser cutting is promoting a change in scientific methodology and is accommodating interdisciplinary approaches. However, the need for knowledge about these technologies and their transfer is increasing at the same time. The SLUB Dresden tries to meet this need by offering an academic makerspa- ce. The successes of this concept and the challenges in its implementation are described here.

Keywords: Makerspace; Fablab; digitalization; academic library; future concept; strategic planning

Inhalt

1 Relevanz von Makerspaces für die Zukunft wissenschaftlicher Bibliotheken . . . . . . . . 70

2 Der SLUB Makerspace . . . . . . . . . . . 71

2.1 Technische Ausstattung . . . . . . . . . . . 71

2.2 Nutzungskonzept . . . . . . . . . . . . 72

2.3 Organisation und Verwaltung . . . . . . . . . 72

2.4 Wo weniger Bücher stehen, ist Platz für

Maschinen ................... 73

3 Veranstaltungen und Wissensvermittlung. . . . . 73

3.1 Niedrigschwelliger Einstieg: Die Offene Werkstatt 73

3.2 Modulare Wissensvermittlung: Die Geräteführerscheine .................. 73

3.3 Individuelle Beratung in der Wissensbar. . . . . . 75

3.4 Zusatzangebote und Sonderveranstaltungen . . . 75

4 Kooperationen und Netzwerk . . . . . . . . . . 76

4.1 Wissenschaftlicher Beirat. . . . . . . . . . 76

4.2 Smart ${ }^{3}$ Academy - Ein aktuelles Beispiel für

Kooperation ................... 76

5 Erfolge des Konzeptes . . . . . . . . . . . . . . 77

5.1 Innovation und kreatives Moment . . . . . . . . 77

5.2 Nachfrage bei Nutzern . . . . . . . . . . . . . . . 78

5.3 Dauerhafte Wirkung: Nutznießer des

SLUB-Makerspace . . . . . . . . . . . . . . . 79

5.4 Die Makerspace-Strategie der SLUB ist

übertragbar . . . . . . . . . . . . 79

6 Herausforderungen und Ausblick. . . . . . . . 80

\footnotetext{
*Kontaktpersonen: Jonas Tiepmar, jonas.tiepmar@slub-dresden.de Jens Mittelbach, jens.mittelbach@slub-dresden.de Melanie Kaiser, melanie.kaiser@slub-dresden.de Daniela Dobeleit, daniela.dobeleit@slub-dresden.de Paul Schwanse, paul.schwanse@slub-dresden.de Uta Fröhner, uta.froehner@slub-dresden.de Maik Jähne, maik.jaehne@slub-dresden.de
} 


\section{Relevanz von Makerspaces für die Zukunft wissenschaftlicher Bibliotheken}

In öffentlichen Bibliotheken sind Makerspaces auch in Deutschland schon länger präsent. So hat sich seit 2013 in der Stadtbibliothek Köln in beispielhafter Weise ein Makerspace etabliert, ${ }^{1}$ in dessen Einrichtung sich moderne Ideen des pädagogischen Diskurses, Konstruktivismus, Flexibilisierung und lebenslanges Lernen manifestiert. ${ }^{2}$ In öffentlichen Bibliotheken ist das Angebot von Makerspaces allerdings in erster Linie als Reaktion auf umfassendere Bedürfnisse der Nutzer hinsichtlich ihrer Freizeitgestaltung $\mathrm{zu}$ verstehen. Hier unterscheiden sie sich von wissenschaftlichen Bibliotheken, in denen der Aspekt der wissenschaftlichen Relevanz von Serviceangeboten eine wichtige Rolle spielt. Gerade in wissenschaftlichen Bibliotheken erschließen Makerspaces zunehmend bedeutungsvolle neue Dimensionen der Wissensarbeit. Zunächst jedoch ist $\mathrm{zu}$ konstatieren, dass nicht alle, weder Bibliotheksmitarbeiter noch Nutzer, auf den ersten Blick erkennen, was eine Werkstatt, in der 3D-Drucker, Lasercutter, aber auch Schleif-, Fräs- und Bohrmaschinen benutzt werden, mit ihrer Universitätsbibliothek zu tun hat. Geprägt vom klassischen Konzept der Bibliothek als einem Speicherort des Wissens, das nach wie vor sowohl in der Wissenschafts- als auch in der Populärkultur virulent ist und perpetuiert wird, verbinden viele Menschen mit dem Begriff Bibliothek Assoziationen von langen Regalreihen, grünen Schreibtischleuchten und Schweigen gebietenden Bibliothekaren. Dabei ist schon in diesem traditionellen Bild der Gedanke des Makerspace angelegt: Bibliotheken waren schon immer ein Ort des Machens. Das Recherchieren nach Informationen, das Bilden von Ideen, das Verfertigen von Gedanken- und Argumentationsfolgen und das Schmieden dieses geistigen Materials zu Texten, die, einmal gedruckt und publiziert, den wissenschaftlichen Zyklus neu speisen und die Wissensbildung neu anregen, wohnt natürlich auch ein handwerklicher Aspekt inne. Und umgekehrt hat das Handwerk des Wissens immer schon eine den Herstellungsprozess transzendierende Bedeutung, die es dem profanen Utilitarismus enthebt. Mit der Digitalisierung und der damit einhergehenden technologischen Revolution verschwinden die Grenzen der früher deutlich getrennten internen und externen Anteile der publizistischen Produktionskette. Doch damit nicht ge-

1 Vgl. Petzold (2016) 287.

2 Vgl. Schuldt (2017) 2. nug. Ähnlich wie die Einführung von Computertechnik, Laserdruck und digitalem Publizieren die Herstellung textueller Objekte verändert hat, befördert das Aufkommen von 3D-Druck und Lasercutting, die Verfügbarkeit von Software zur 3D-Modellierung und von VR-Brillen den Methodenwandel in der Wissenschaft und kommt interdisziplinären Ansätzen entgegen. ${ }^{3}$ Nicht nur Materialwissenschaftler, Maschinenbauer und Architekten profitieren vom neuartigen digitalen Prototyping, auch Medizinern und Biologen, Archäologen und Historikern eröffnen sich bislang ungeahnte Möglichkeiten durch die Verfügbarkeit solcher Infrastrukturen. Indem sich aber das Methodenwissen durch komplexe Technologien auffächert, wachsen die Herausforderungen, diese Technologien zu meistern und die entsprechenden Methoden virtuos einzusetzen. Bibliotheken, Orte der Wissensgenerierung, so zu entwickeln, dass ihre Benutzer in die Lage versetzt werden, mit den Entwicklungen Schritt zu halten und durch Learning by Doing Methodenkompetenz aufzubauen, ist folgerichtig. Wissen manifestiert sich im digitalen Zeitalter längst nicht mehr nur in Büchern bzw. Texten; vielmehr gewinnt es mit Modellbau und Rapid Prototyping, mit 3DVisualisierung oder digitalen Ton- und Filmobjekten vielfältige neue Ausdrucksformen. Makerspace in wissenschaftlichen Bibliotheken tragen in integrativer Weise zum Auf- und Ausbau von wissenschaftlicher Methodenkompetenz gemäß den Forderungen der Hochschulrektorenkonferenz von 2012 bei. $^{4}$

Diese Erkenntnisse - und intensive Kontakte mit der Dresdner FabLab-Szene - haben die Sächsische Landesbibliothek - Staats- und Universitätsbibliothek Dresden (SLUB) 2014 dazu veranlasst, einen Makerspace aufzubauen, zunächst in einer Projektphase, später bei parallelem Regelbetrieb. Sie ist damit die erste wissenschaftliche Bibliothek in Deutschland, die ihr Service-Angebot um diesen Sektor bereichert hat. Von Anfang an stand die Arbeit des kleinen, aber sehr engagierten Teams, das sich dieser Aufgabe angenommen hat, unter dem Motto „Wissen kommt von Machen“. Dieses Motto traf zunächst einmal vor allem auf die Team-Mitglieder selbst zu, denn für alle war die Beschäftigung mit Technologien und Methoden aus dem FabLab-Bereich und der Maker-Szene Neuland, das nur durch Beschreiten erschlossen werden konnte. Der Wechsel aus klassischen Bibliotheksbereichen darf dabei nicht bedeuten, die Verbindung zu ihnen zu verlieren. Ziel war und ist es, Räume und Dienstleistungen fortlaufend so zu entwickeln, dass Werkzeuge und Methoden nichttextueller

3 Noenning, Oehm und Wiesenhütter (2014) $34 \mathrm{ff}$. 4 Vgl. Hochschulrektorenkonferenz (2013) 9. 
Wissensproduktion und -vermittlung mit den klassischen Angeboten der SLUB dauerhaft sinnvoll verknüpft sind. An der Schnittstelle zwischen Nutzern und Maschinen stehen Daten, deren Erzeugung und Verarbeitung heute in vielfältigster und komplexer Weise geschieht und daher ein hohes Niveau an Know-how sowie fortdauerndes Lernen voraussetzt. Wenngleich die Hürden, die Interessierte am Einstieg ins experimentelle Fertigen hindern, aufgrund des technologischen Fortschritts gesunken sind, ist der Zugang zu technischen, räumlichen und sozialen Infrastrukturen nach wie vor der entscheidende Faktor, der für das einzelne Individuum den Aufbau eines entsprechenden Wissensrepertoires bestimmt. Infrastrukturen bereitzustellen und als Vermittlungsknoten innerhalb von Kompetenznetzwerken zu fungieren, ist die Aufgabe von wissenschaftlichen Bibliotheken, weshalb der neue Servicebaustein Makerspace ohne Brüche in deren Betriebsorganisation passt - mit Zusatznutzen für die Nutzer der SLUB wie auch für die Bibliothek selbst.

Die abteilungsübergreifende „Arbeitsgruppe Makerspace“ sorgt für die Ausgestaltung und Durchsetzung eines stimmigen Konzeptes, das sich in das bibliothekarische Gesamtangebot einfügt. Dabei wird sie unterstützt von einem wissenschaftlichen Beirat aus Professoren der Technischen Universität Dresden (TUD) und SLUB-Mitarbeitern, der die Interessen und Beiträge der Akteure des Universitätscampus und der Partner der Forschungsallianz Dresden-concept e.V. koordiniert. Eine Herausforderung für das Makerspace-Team, die Bibliotheksleitung und die Kooperationspartner war insbesondere die Frage, wie die Wissensvermittlung zu Werkzeugen und Methoden des Prototyping zu konzipieren und zu gewährleisten sei. Wie befähigen wir Nutzer mit sehr unterschiedlichen Wissensständen zur praktischen Arbeit mit den teils hochkomplexen Geräten im Makerspace? Welche organisatorischen Grundbedingungen (Öffnungszeiten, personelle Ausstattung, Zugang) muss das Benutzungskonzept berücksichtigen und welche Arbeitsschutzbedingungen müssen gewährleistet sein? Welche Veranstaltungsformate müssen entwickelt werden, damit sich unsere Nutzer, gemäß unserer Grundidee, zum „Selbermachen“ in der SLUB angeregt fühlen?

\section{Der SLUB Makerspace}

\subsection{Technische Ausstattung}

Neben klassischen Werkzeugen, wie Werkzeugset, Bohrmaschine und Stichsäge, bietet das Makerspace verschiedene Maschinen rund um die digitale (und analoge) Pro- duktion. So können digitale Daten aus CAD-Programmen (Computer Aided Design - Computergestütztes Entwickeln) durch generative Fertigungsverfahren/Urformen wie dem 3D-Druck als auch durch Trennen von Material (Laserschneiden, Styroporschneiden) in physikalische Objekte umgesetzt werden. Nachdem Maschinen für diese Verfahren in den letzten Jahren durch abgelaufene Patente und günstigere elektronische Komponenten stetig im Preis gesunken und für jedermann verfügbar geworden sind, eröffnen diese Maschinen den Nutzern neue, schnelle und kostengünstige Produktionswege. Diese Entwicklung ist ähnlich gravierend wie die Revolution durch Personal Computer. Durch den Preisverfall und technologische Fortschritte wird 3D-Druck nicht mehr nur bei der Entwicklung von Prototypen eingesetzt, sondern kommt heutzutage zum Beispiel bei Automobilherstellern in der Serienproduktion zum Einsatz..$^{5}$ Auch in Design- und Architekturbüros, Ingenieurbüros und vielen anderen Bereichen werden 3D-Drucker täglich eingesetzt. Somit ist das Erlernen und die Anwendung dieser Produktionstechniken nicht mehr nur etwas für Enthusiasten oder „Nerds“, sondern gehört schon heute zum Alltag, nicht nur in ingenieurswissenschaftlichen Fächern.

Der SLUB Makerspace bietet mit seinem Maschinenund Wissensangebot eine fortschrittliche Lernwerkstatt, in der theoretisches Wissen und digitale Daten in physikalische Objekte übersetzt werden. Der Maschinenpark des SLUB Makerspace umfasst: ${ }^{6}$

- Kleingeräte(Bohrmaschine, Stichsäge, Dremel, Schleifband u.a.)

- Geräte zur Kunststoffbearbeitung (Tiefziehmaschine, Kunststoffbiegemaschine)

- Styroporschneider, 4-achsig (für 3D Schnitte geeignet)

- 3D-Drucker (Verfahren: Binder Jetting, FDM - Fused Deposition Modeling - Schmelzschichtverfahren)

- Lasercutter (40 Watt, Bearbeitungsfläche ca. 900 x $600 \mathrm{~mm}$ )

- Elektronikarbeitsplatz (Lötstation, Heißluftlöten, Oszilloskop u.a.)

Neben dem großen Maschinenpark gibt es noch diverse Lehrmaterialien, die dabei helfen, digitale Technologien praktisch zu erlernen. Es können Arduino-Kits ausgeliehen werden, mit deren enthaltenen Sensoren und Aktoren man Objekten, die mithilfe von Lasercutter und 3D-Drucker gebaut wurden, Leben einhauchen kann. 3D-Brillen bieten

5 Vgl. Zarre (2017) und Gartner (2012).

6 Dies ist nur eine Auswahl. Alle Geräte sind gelistet bei Dresden Technologieportal (2017). 
die Möglichkeit, schon vor deren Fertigung mit Objekten zu interagieren. Neben der klassischen Werkstatt stellt die SLUB inzwischen systematisch weitere Angebote unter dem Begriff Makerspace zur Verfügung: So ist von der Abteilung Musik, Referat Mediathek, ein Ton- und Videostudio eingerichtet worden (Makerspace M3) und steht Nutzern für die Produktion von Multimediainhalten zur Verfügung.

\subsection{Nutzungskonzept}

Die Räume und Geräte des SLUB-Makerspace stehen allen Nutzern der SLUB zur Verfügung. Voraussetzung zur Nutzung der Maschinen und Geräte im Makerspace ist eine gültige Arbeitsschutzunterweisung und - so erforderlich ein Geräteführerschein für das jeweilige Gerät oder die Gerätegruppe (z. B. Kleingeräte). Während Kleingeräte ohne vorherige Reservierung ausgeliehen und vor Ort benutzt werden können, müssen Großgeräte reserviert werden. Hierzu wurde das Raumbuchungssystem der SLUB, ${ }^{7}$ welches auf dem Open-Souce-Tool MBRS basiert, ${ }^{8}$ an die Bedürfnisse des Makerspace angepasst: Geräte entsprechen virtuellen Räumen und - im Gegensatz zu den restlichen Räumen in der SLUB - sind diese virtuellen Räume nur durch autorisierte Personen buchbar. Hiermit soll sichergestellt werden, dass die Geräteressourcen fair verteilt werden, möglichst vielen Leuten zur Verfügung stehen und die Zeiten sinnvoll genutzt werden. Auch ist eine Vorabkontrolle des gebuchten Termins hinsichtlich Verfügbarkeit und Plausibilität möglich.

Nutzer schicken ein Formular ab, über das neben Nutzernummer und E-Mail-Adresse Wunschtermin (aus dem Raumbuchungssystem sind die freien Termine ersichtlich), Gerätetyp, Material, geschätzte Dauer und eine Auftragsbeschreibung übermittelt werden können. Außerdem kann eine Datei angehängt werden. Die Mitarbeiter im Makerspace-Team nehmen die entsprechende Gerätebuchung vor. Wird eine Datei mitgeschickt, wird diese manuell auf Fehler geprüft (bei 3D-Druck: Wasserdichte Modelle, nicht druckbare Details, Zeitdauer; bei Lasercutter: Abschätzung Bearbeitungszeit). Für Einsteiger ist es oft schwer, die Dauer eines 3D-Drucks abzuschätzen, eine häufige Korrektur seitens des Makerspace-Teams ist dadurch die Anpassung der Bearbeitungsdauer und somit die Anpassung des gebuchten Termins, diese Maßnahmen reduzieren nicht nur das Konfliktpotential im Regel-

7 SLUB Dresden (2017c).

8 Morrison und Beranek (2017). betrieb, sie erleichtern dein Einstieg in die Nutzung der angebotenen Geräte und Technologien. Nach dieser Prüfung und einer eventuell notwendigen Anpassung erfolgt eine erneute Bestätigung oder eine Absage des Termins durch den Nutzer. Besitzen die Nutzer einen Geräteführerschein für das Gerät, kann nun ohne individuelle Betreuung am Gerät gearbeitet werden, die Mitarbeiter stehen allerdings weiterhin für Rückfragen vor Ort zur Verfügung. Hat die Person noch keinen Geräteführerschein, werden die Maschinen und Geräte durch SLUB-Mitarbeiter bedient, während die Nutzer durch Zuschauen lernen können.

Den Nutzern wird bei Buchung des Termins ein Hinweis mit den für das gebuchte Gerät gültigen spezifischen Parametern geschickt. Unsere 3D-Drucker bieten je nach Modell verschieden große Bauräume, die die Größe des zu fertigenden Objektes begrenzen. Selbst grundlegende Tipps zur Dateierstellung werden mitgeschickt. Dies ermöglicht den Nutzern die Vorbereitung ihrer Dateien, um dies nicht erst vor Ort erledigen zu müssen. Durch Einführungskurse, Präsentationen und Beratung vor Ort wird den Nutzern bei der Erstellung von Daten geholfen, was in der Regel dankbar in Anspruch genommen wird.

\subsection{Organisation und Verwaltung}

Abgerechnet wird die Maschinenlaufzeit im 20-MinutenTakt (wobei jeder Takt einer „Gebührenstufe“ im Bibliothekssystem LIBERO entspricht). Die Kosten sind auf der Webseite des Makerspace bei der jeweiligen Maschine aufgelistet. ${ }^{9}$ Eine Ausnahme bildet der Lasercutter, hier sind die ersten zehn Minuten Maschinenlaufzeit frei, Kosten werden erst danach fällig. Bei den 3D-Druckern sind die Verbrauchsmaterialien schon enthalten, bei Nutzung des Lasercutters muss eigenes Material mitgebracht werden. Kleingeräte sowie Geräte ohne lange Laufzeiten (Kunststoffbiegemaschine, Tiefziehmaschine) stehen kostenfrei zur Verfügung. Die Gebührenordnung ist dabei nicht ökonomisch motiviert, um die verursachten Kosten zu decken oder einen Gewinn zu erwirtschaften. Die Gründe für die eingeführte Gebührenordnung liegen hauptsächlich in der Notwendigkeit der Organisation und Regulierung der Nachfrage. Die faire Verteilung von Maschinenzeiten auf Nutzer ist eine wichtige Bedingung, die über die Erhebung moderater Gebühren unterstützt werden kann. Durch anfallende Gebühren werden Nutzer stimuliert, Maschinenzeiten nicht vorsorglich, leichtfertig oder im Übermaß zu

9 SLUB Dresden (2017a). 
buchen. Die anfallenden Kosten lassen die Nutzer ihre Projekte darüber hinaus intensiver und überlegter vorbereiten, so dass der Lerneffekt gefördert wird.

\subsection{Wo weniger Bücher stehen, ist Platz für Maschinen}

Digitales Publizieren schont nicht nur die Umwelt, es hinterlässt in Bibliotheken leere Räume. Einer dieser Räume, der ehemalige Atlantenlesesaal in der Bereichsbibliothek DrePunct der SLUB Dresden, wird seit 2014 als Makerspace M1 genutzt, hier finden neben den aufgeführten Geräten (vgl. Abschnitt 2.1 Technische Ausstattung) noch Tische, Stühle und Präsentationstechnik Platz - so können Workshops bzw. Unterrichtseinheiten, aber auch Entwicklungstätigkeit in einer kreativen Atmosphäre stattfinden - mit direktem Zugriff auf die Maschinen. Durch die große Nachfrage nach weiteren Arbeitsräumen werden in der Bereichsbibliothek DrePunct, in unmittelbarer Nähe zum Makerspace M1 weitere Räume zur Verfügung gestellt, die unterschiedliche Nutzeranforderungen bedienen: ein Gruppenarbeitsraum für größere Gruppen und Seminare (M2) und verschiedene Carrels (Räume für Kleingruppen).

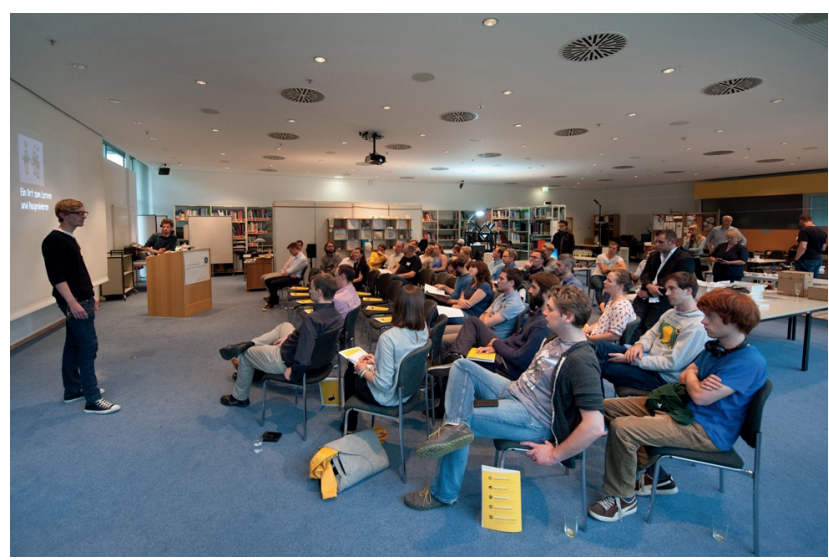

Abb. 1: Lehrveranstaltung im Makerspace M1

\section{Veranstaltungen und Wissensvermittlung}

Das Konzept zur Wissensvermittlung im Makerspace ist integraler Bestandteil des Gesamtkonzeptes der SLUB. Ziel ist der Aufbau eines Do-It-Yourself-Betriebes. Während zu Anfang Individualbetreuung eines jeden Makers an den Geräten notwendig ist, befähigen die Geräteführerscheine die erfahreneren Nutzer, ihre Projekte selbstständig an den
Geräten zu realisieren. Eine Betreuungsperson aus dem Makerspace-Team leistet bei konkreten Fragen Hilfestellung - so wie an den Informationspunkten in Bibliotheken Unterstützung bei der eigenständigen Recherche angeboten wird. Sehr komplexe Anfragen, die über ein Basisniveau hinausgehen, werden über die Wissensbar an die jeweiligen Experten weitergeleitet.

\subsection{Niedrigschwelliger Einstieg: Die Offene Werkstatt}

Eine wesentliche Herausforderung bei der Anwendung und Bereitstellung von innovativen Technologien ist die relativ hohe Anforderung an die Vorkenntnisse der interessierten Person. Je nach Erfahrung und Hintergrund variieren diese Vorkenntnisse stark. Die Mitarbeiter im Makerspace versuchen, die Einstiegshürden für Interessierte so niedrig wie möglich zu halten. Dennoch bleibt die Lernkurve bei komplexen Technologien anfangs recht steil. Deshalb gilt es, Nutzer zu ermutigen, sich der daraus ergebenden Herausforderung zu stellen. Erreicht wird das durch verschiedene einführende Veranstaltungen wie Schülerführungen, Summer Schools oder die Lange Nacht der Wissenschaften, an der der SLUB Makerspace regelmäßig teilnimmt. ${ }^{10}$

Aber auch im Regelbetrieb sind Angebote erforderlich, die einführenden Charakter haben. Ein Beispiel dafür ist die Offene Werkstatt, die mehrmals wöchentlich stattfindet. In der Offenen Werkstatt können die Nutzer den Makerspace kennenlernen und kleinere Projekte realisieren; sie können Arbeiten für den Lasercutter, die nicht länger als zehn Minuten dauern, bearbeiten. Das Team Makerspace steht dabei für Fragen rund um das Arbeiten und die Technologien im Makerspace zur Verfügung. Außerdem ist der Werkstatt-Raum M1 für freies Arbeiten mit Kleingeräten an den Werkbänken, für Nacharbeiten an Werkstücken oder für die Gruppenarbeit im Sitzbereich nutzbar.

\subsection{Modulare Wissensvermittlung: Die Geräteführerscheine}

Grundlage des modularen Kursangebotes ist die allgemeine Arbeitsschutzunterweisung und der Geräteführerschein Kleingeräte, die beide gemeinsam in einem Kurs angeboten werden. In einer Vor-Ort-Schulung weist der Referent

10 Vgl. z.B. Krzywinski (2014), Kaiser (2016), Kaiser und Fröhner (2016). 


\section{SLUB}

MAKER

SPACE

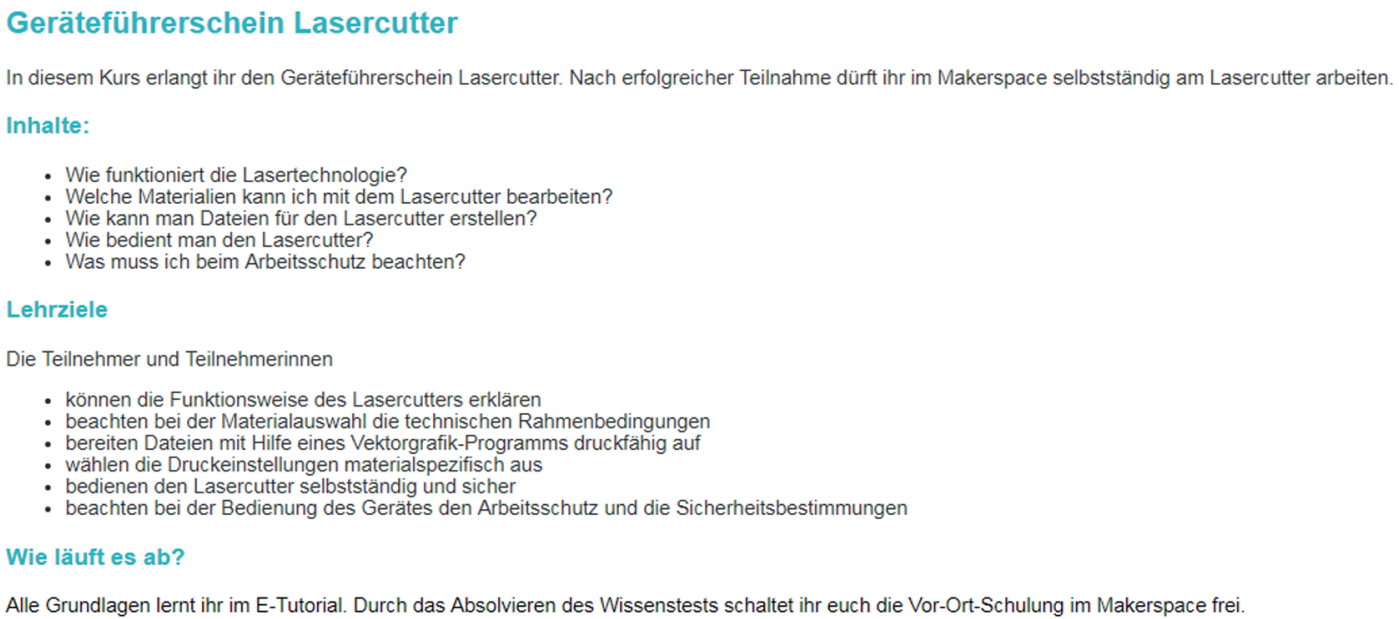

Alle Grundlagen lernt ihr im E-Tutorial. Durch das Absolvieren des Wissenstests schaltet ihr euch die Vor-Ort-Schulung im Makerspace frei.

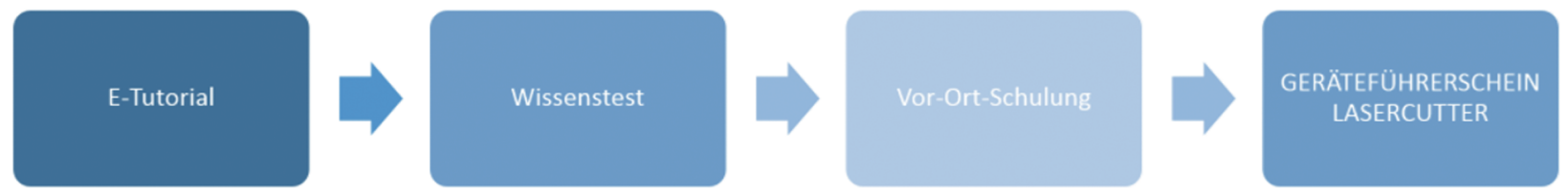

>>Hier geht es los: Start des E-Tutorials!

» Ihr habt schon Erfahrungen mit dem Lasercutten? Dann geht gleich zum Test und stellt euer Wissen unter Beweis!

Ansprechpartner

Team Makerspace-makerspace@slub-dresden.de

Abb. 2: Geräteführerschein Lasercutter auf OPAL von Daniela Dobeleit/SLUB Dresden ist lizenziert unter einer Creative Commons Namensnennung 4.0 International Lizenz.

die Teilnehmer in die Benutzung der Kleingeräte (Dremel, Styroporcutter, Akkuschrauber, Kunststoffbiegemaschine usw.) ein und informiert sie über die Verhaltensregeln im Gefahrenfall. Die Belehrung muss von den Teilnehmern unterzeichnet werden und wird im Benutzerkonto vermerkt. Ohne diesen mit der Arbeitsschutzunterweisung kombinierten Geräteführerschein ist ein selbstständiges Arbeiten im Makerspace oder der Erwerb von anderen Geräteführerscheinen nicht möglich. Die Arbeitsschutzbelehrung wird aus arbeitsschutzrechtlichen Gründen alle sechs Monate erneuert.

Bei Interesse können die Nutzer den Geräteführerschein 3D-Kunststoffdruck und Lasercutter absolvieren. Diese Kurse entwickelte das Team im Blended-LearningDesign. Nach dem Inverted-Classroom-Modell durchlaufen die Teilnehmer ein E-Tutorial, welches mit einem Wissenstest abgeschlossen werden muss. Das Bestehen des Testes ist Voraussetzung für die Teilnahme an der folgenden Vor-Ort-Schulung im Makerspace:
„Die Grundidee des Inverted Classroom Model ist es, die Inhaltsvermittlung, die traditionell gemeinsam vor Ort mit dem Lehrer stattfindet, und das Üben und Vertiefen, das zu Hause allein erledigt wird, zu vertauschen. Das Ziel dabei ist es, Zeit für das gemeinsame Lernen und das Anwenden des neu Gelernten zur Verfügung zu haben.“"11

Das Niveau der Teilnehmer hinsichtlich ihrer Fachkultur, ihres individuellen Vorwissens sowie ihrer Erfahrungen mit Technik ist sehr heterogen. Durch die Vermittlung von Grundlagenwissen im E-Tutorial (vgl. Abb. 2) werden diese unterschiedlichen Wissensstände aufgefangen. Während Einsteiger sich niedrigschwellig mit der Thematik auseinandersetzen, ist es erfahrenen Teilnehmer möglich, sofort den Test zu absolvieren und zu der Vor-Ort-Schulung zugelassen zu werden. In diesem Praxismodul weisen die Referenten die Teilnehmer in die praktische Bedienung der

11 Schäfer (2012) 3. 
Geräte ein und vertiefen das erlernte Grundlagenwissen durch Übungen und Praxisbeispiele. Im Geräteführerschein Lasercutter werden im E-Tutorial beispielsweise die möglichen Materialien, die Software-Einstellungen und die Vorbereitung der Datei behandelt. In der Vor-Ort-Schulung nehmen die Teilnehmer gemeinsam den Lasercutter in Betrieb und erhalten eine Testdatei, die sie durch die Bereinigung von Fehlern für die Bearbeitung optimieren müssen. Danach werden unterschiedliche Proben mit unterschiedlichen Materialien geschnitten oder graviert, um die Teilnehmer mit den Eigenheiten der Materialien und ihren Grenzen vertraut zu machen.

Der Erwerb neuen Wissens und neuer Fähigkeiten wird zusätzlich durch die Veranschaulichung abstrakter Inhalte unterstützt. Um verschiedenen Lerntypen entgegen zu kommen, nutzt das Team verschiedene Medienarten im E-Tutorial. Neben Texten und Tabellen sind komplexe Sachverhalte mittels Abbildungen und Videos anschaulich aufbereitet. Außerdem stehen Checklisten und Materiallisten mit Parametern zur Verfügung, die die Maker bei der Realisierung ihrer Projekte zur Hand nehmen können. Einige Erklärvideos produzierte das Team mit der webbasierten Software GoAnimate ${ }^{12}$ selbst. Die Hauptaussagen der Erklärfilme bettete das Team in Geschichten aus der Realität verschiedener Nutzertypen ein. Durch das „Storytelling“ bleiben die Inhalte den Rezipienten besser in Erinnerung und der Praxistransfer des Wissens wird erleichtert. Außerdem können sich die Nutzer mit den Protagonisten identifizieren, etwa mit „Alex“, der sein Praxissemester an einer Forschungseinrichtung absolviert und die Vorstellung eines Prototyps vorbereiten muss (vgl. Abb. 3).

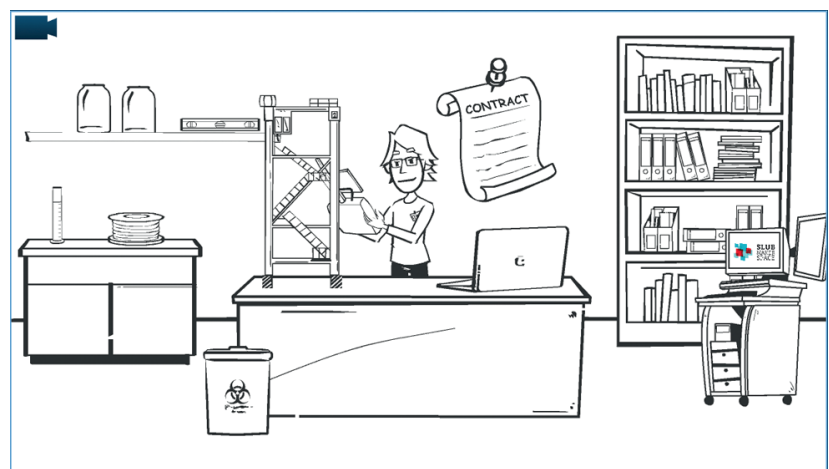

Abb. 3: Auszug aus SLUBcast - Welche Materialien kann ich mit dem Lasercutter bearbeiten? von Daniela Dobeleit / SLUB Dresden lizenziert unter einer Creative Commons Namensnennung 3.0 Deutschland Lizenz

12 GoAnimate (2017).
Auch gut aufbereitete Youtube-Videos und Filme aus der Datenbank Lynda.com (früher video2brain) ${ }^{13}$ wurden in die E-Tutorials eingebettet, welche neben dem didaktischen Nutzen gleichzeitig die Verbindung zum SLUB-Bestand herstellen.

Die Blended-Learning-Kurse veröffentlicht das Makerspace-Team zum Start des WS 2017/2018 unter einer Creative Commons Namensnennung 3.0 Deutschland Lizenz als Open Educational Resources (OER) auf der Lernplattform OPAL. Die Unterlagen und Videos stehen für die Nachnutzung und Weiterentwicklung zur Verfügung. Neben den Geräteführerscheinen Kleingeräte, Lasercutter und 3D-Kunststoffdruck wird das Team noch Kurse zum Styroporcutter und Elektronikarbeitsplatz entwickeln. Es ist zukünftig geplant, Aufbaumodule für die Geräte anzubieten, beispielsweise ein Modul zur Stempelherstellung oder zum Gravieren von Gläsern und Flaschen als Ergänzung zum Geräteführerschein Lasercutter. Durch den Einsatz von Gamification-Elementen, wie Highscores und Rankings, sollen die Nutzer zum Erwerb möglichst vieler Module motiviert werden.

\subsection{Individuelle Beratung in der Wissensbar}

Nutzer profitieren neben den Kursen, die im öffentlichen Veranstaltungskalender der Bibliothek beworben werden, von der Individualberatung in unserer im Jahr 2014 preisgekrönten Wissensbar, einer modernen Form der individuellen Beratung. ${ }^{14}$ Während der Öffnungszeiten ist bei speziellen Fragen zum eigenen Modell oder zu Fertigungstechniken nicht immer der richtige Ansprechpartner vor Ort oder die Zeit der Betreuungsperson ist aufgrund von Parallelterminen knapp bemessen. Für solch komplexe Anfragen stehen die Experten des Teams in der Wissensbar zur Verfügung. Die Maker haben die Möglichkeit, hier kostenfreie Beratungstermine in Anspruch zu nehmen. Das Team berät eingehend zur 2D- und 3D-Modellierung, zur Modellaufbereitung für den Lasercutter und zum 3DKunststoffdruck.

\subsection{Zusatzangebote und Sonderveranstaltungen}

Einen Rahmen um die Basisschulungen bilden die regulären und einmaligen Sonderveranstaltungen. Mit dem Ma-

13 Lynda.com (2017).

14 Vgl. Mittelbach und Muschalek (2015) sowie DBV (2014). 
kerspace werden zudem zusätzliche Veranstaltungsformate in der SLUB erprobt. Während des Semesters findet jeden ersten Donnerstag im Monat das SLUB Makerspace Meet up! statt. Ziel dieser Zusammenkünfte ist es, mit den Teilnehmern in einer lockeren und ungezwungenen Atmosphäre über Technologien und Werkzeuge des Makerspace $\mathrm{zu}$ sprechen und Anknüpfungspunkte für gemeinsame Vorhaben und Workshops zu finden. Um ein möglichst breites Publikum anzusprechen, ist eine Teilnahme ohne Vorkenntnisse möglich. Jedes Meet up! hat ein eigenes Thema und besteht aus dem Vortrag eines Experten zu dem gewählten Thema und einer anschließenden Erprobungsphase verschiedener Beispielanwendungen. Durch das breite Themenspektrum werden neue Zielgruppen erschlossen und das Community-Building unterstützt, da im Anschluss an die Veranstaltung genügend Zeit für Diskurs und Austausch eingeplant wird. Bei einem Meet up! zum Thema 3D-Modellierung fanden sich Interessierte zum Thema 3D-Modellierung mit Blender (einer freien 3D-Grafiksuite) zusammen und bildeten einen Stammtisch. Aus diesem Stammtisch heraus entwickelte sich ein Grundlagenkurs, welcher regelmäßig, organisiert durch freiwillige Referenten aus der Community und dem MakerspaceTeam, stattfindet.

\section{Kooperationen und Netzwerk}

\subsection{Wissenschaftlicher Beirat}

Der SLUB Makerspace pflegt eine enge Beziehung zu verschiedenen Professuren der TU Dresden, Forschungsinstituten und zu Einrichtungen aus der sog. Maker-Szene. Der manifestierte Bestandteil des Netzwerkes ist dabei der wissenschaftliche Beirat des Makerspace, diesem Beirat gehören unteranderem an:

- Jun.-Prof. Dr.-Ing. Jens Krzywinski, Juniorprofessur für Technisches Design, Institut für Maschinenelemente und Maschinenkonstruktion, TU Dresden

- Prof. Dr.-Ing. Daniel Lordick, Professur für Geometrie, AG Geometrische Modellierung und Visualisierung, Institut für Geometrie, TU Dresden

- Prof. Dr.-Ing. Jörg Rainer Noennig, Professur für Wissensarchitektur - Laboratory of Knowledge Architecture, Institut für Gebäudelehre und Entwerfen, TU Dresden

- Fraunhofer-Institut für Werkzeugmaschinen und Umformtechnik (IWU)

Einzelne Partner stellen Werkzeuge und Technik in Form von Dauerleihgaben zur Verfügung und öffnen so deren
Nutzung einem breiteren Publikum. Konkret waren der erste Lasercutter und ein professioneller 3D-Drucker der AG Geometrische Modellierung und Visualisierung die ersten Geräte welche der SLUB Makerspace 2015 bereitstellen konnte.

Darüber hinaus bestehen Beziehungen zu verschiedenen Lehrstühlen der TU Dresden wie der Professur für Verarbeitungsmaschinen und Verarbeitungstechnik oder der Professur für Ingenieurpsychologie. Auch die Verknüpfung mit der regionalen Maker-Szene ist sehr gut ausgebaut. Ein Beispiel sind die engen Beziehungen zum FabLab Dresden (Konglomerat e.V.), mit dem wechselseitig Kurse veranstaltet werden, um den jeweiligen Nutzern einen Einblick in alternative Räumlichkeiten, technische Ausstattungen und Werkstattkonzepte zu geben. Weiterhin nutzen Communities wie der beschriebene BlenderStammtisch unsere Angebote. Es gibt Kooperationen zu Projekten wie E3P (3D-Drucker für Schulen) oder Elbflorace (Das Dresdner Formula Student Team). Kern dieser Kollaborationsbeziehungen ist nicht nur die Bereitstellung von Angeboten in Form von Räumen oder technischer Ausrüstung, sondern auch die Interaktion mit uns oder mit anderen Nutzern zum Wissensaustausch.

\subsection{Smart $^{3}$ Academy - Ein aktuelles Beispiel für Kooperation}

Gemeinsam mit dem Fraunhofer-Institut für Werkzeugmaschinen und Umformtechnik entwickelt das Makerspace-Team gegenwärtig ein neues Veranstaltungskonzept die Smart ${ }^{3}$ Academy. Eine Veranstaltungsreihe über ein gesamtes Semester in Zusammenarbeit mit dem Netzwerk Smart ${ }^{3}$. Rund um das Thema Intelligente Materialien werden alle zwei Wochen spannende Themen präsentiert: von methodischer Ideenfindung über verschiedene 3D-DruckTechnologien bis zur angeleiteten Prototypenfertigung. Die nichttextuelle Wissensvermittlung und die praktische Anwendung des erlangten Wissens stehen auch bei dieser Veranstaltung im Vordergrund. Dabei soll die didaktische geleitete, wechselnd stattfindende Mischung aus Vermittlung theoretischer Grundlagen zu den genannten Schwerpunkten und praktischer Unterstützung bei der Umsetzung eigener Ideen durch die Betreuung der Arbeitsphasen ein bestmöglichen Austausch von neuen innovativen Ideen und bereits vorhandenen Erfahrungen ermöglichen. Spezielle Literatur und relevante Materialdatenbanken werden dabei an einem eigens für die Veranstaltung eingerichteten Arbeitsplatz zur Verfügung gestellt. Der Smart Workspace wird in Kooperation mit Designern, dem IWU, sowie Nutzern des Makerspace entwickelt. Ein Bereich innerhalb des 
Makerspace, der zusätzlich zu den genannten Ausstattungen noch einen Arbeitsplatz, Werkzeuge und themenspezifische Experimente bietet.

Während der Entwicklung technischer Prototypen ist die Dokumentation von Erkenntnissen und getroffenen Entscheidungen von höchster Bedeutung. Um den Teilnehmern der Smart ${ }^{3}$ Acadamy die Dokumentationsarbeit ihres Projektes zu erleichtern, wird die Veranstaltung mit einem „Smart Blog \& Write“-Seminar ergänzt. Das Netzwerk Schreiben der SLUB bietet den Teilnehmern dazu in diesem Veranstaltungsbaustein einen Einblick in das Bloggen wissenschaftlicher Texte und Ergebnisse und begleitet sie bei der Dokumentation ihres Projektes. ${ }^{15}$ Die Professur für Wissensarchitektur bindet diese Veranstaltung in Ihre Lehrveranstaltungen ein und leistet einen großen Anteil bei der Präsentation verschiedener Themen. Neben der Teilnahme an der entsprechenden Lehrveranstaltung sind die Verwirklichung eigener Projektideen und die Weiterbildung im Bereich intelligente Materialien wesentliche Motivation für eine Teilnahme an dieser Veranstaltung. Um weitere Anreize für Nutzer zu setzen, wird einer der entstandenen Prototypen zum Abschluss der Veranstaltung mit dem Smart ${ }^{3}$ Academy Award ausgezeichnet.

Ebenfalls ein sehr produktiver und aktueller Kooperationsbereich ist die Zusammenarbeit mit dem Landesamt für Archäologie (Sachsen). Im Gegensatz zur Smart ${ }^{3}$ Academy als gemeinsam konzipierte Veranstaltung ist diese Zusammenarbeit geprägt von der gegenseitigen Nutzung technischer Ausstattung. Zum einen nutzen die Kollegen die technischen Möglichkeiten des SLUB Makerspace (insbesondere den 3D-Druck) zur Herstellung von vergrößerten Anschauungsobjekten; zum anderen greift der Makerspace auf die 3D-Scan-Technik des Landesamtes für Archäologie zurück, um in eigenen Beständen zu experimentieren (Welche Möglichkeiten bietet zum Beispiel der 3D-Scan im Bereich Archiv oder Buchmuseum?).

Ein Beispiel für etablierte Veranstaltungskonzepte ist die Construction Bionics Summer School. Dort treffen Studenten und Wissenschaftler aus verschiedensten Ländern aufeinander und entwerfen in Kleingruppen innerhalb von fünf Tagen neue Ideen zur Nutzung von Bionik im Kontext der Architektur.

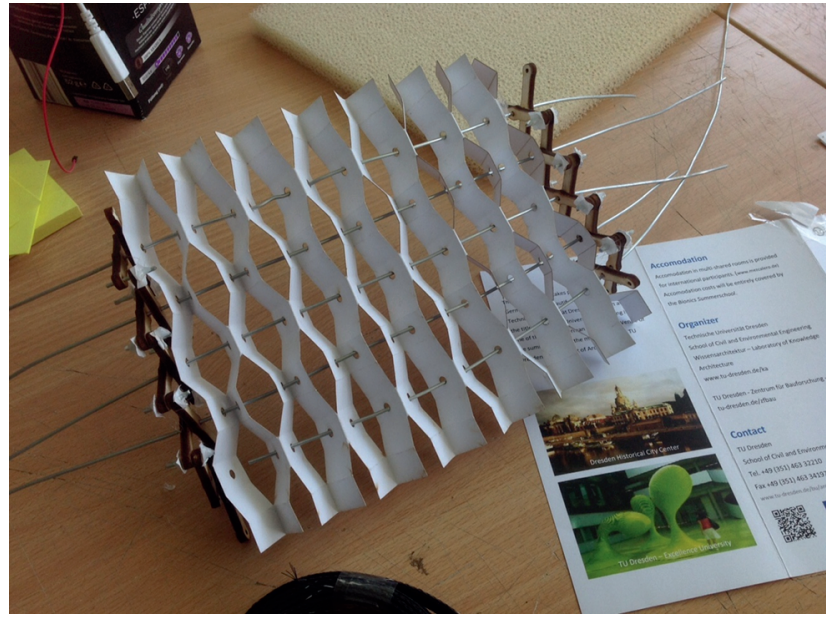

Abb. 4: Modell eines faltbaren, Origami inspiriertem, multifunktionalem Wandmodul

Abschließend sind die Beiträge des SLUB Makerspace auf den zahlreichen Messen der Maker-Szene (bspw. Maker Fair, Maker Con usw.) zu nennen, die ebenfalls einen wichtigen Bestandteil für die Vernetzung und Kommunikation mit Interessierten und Partnern darstellen.

\section{Erfolge des Konzeptes}

\subsection{Innovation und kreatives Moment}

Wie sich gezeigt hat, besteht ein guter Teil der Innovation des Makerspace in der Zusammenarbeit mit zahlreichen Partnern der Dresdner Wissenschaftslandschaft und der sog. Kreativwirtschaft. Diese Kooperationen haben zum einen die Markteinführung des neuen Servicebausteins Makerspace erst ermöglicht, zum anderen bieten sie der Bibliothek finanziell, personell und materiell Vorteile durch die Schaffung von Personalstellen und die Überlassung von Geräten und Dauerleihgaben. Mit der Bündelung der Technikressourcen erhöht sich für die SLUB die Akzeptanz auf dem Campus und in den Gremien der Universität, auch die Attraktivität für potentielle Nutzer sowie neue Partner wird gesteigert. Die Partner der SLUB profitieren ihrerseits von den genannten Veranstaltungsreihen und der oben aufgeführten kreativen Verbindung klassischer Bibliotheksressourcen mit der „,neuen Welt“ durch: Geräteführerscheine mit Kursen, Literatur für Projekte, Werkzeuge und Werkstoffe (z. B. smart materials), Nutzung vorhandener Bibliotheks- und Informationsinfrastruktur (z. B. LIBERO für die Nutzerverwaltung oder Raumbuchungssoftware für die Geräteverwaltung und Reservierungen). 


\subsection{Nachfrage bei Nutzern}

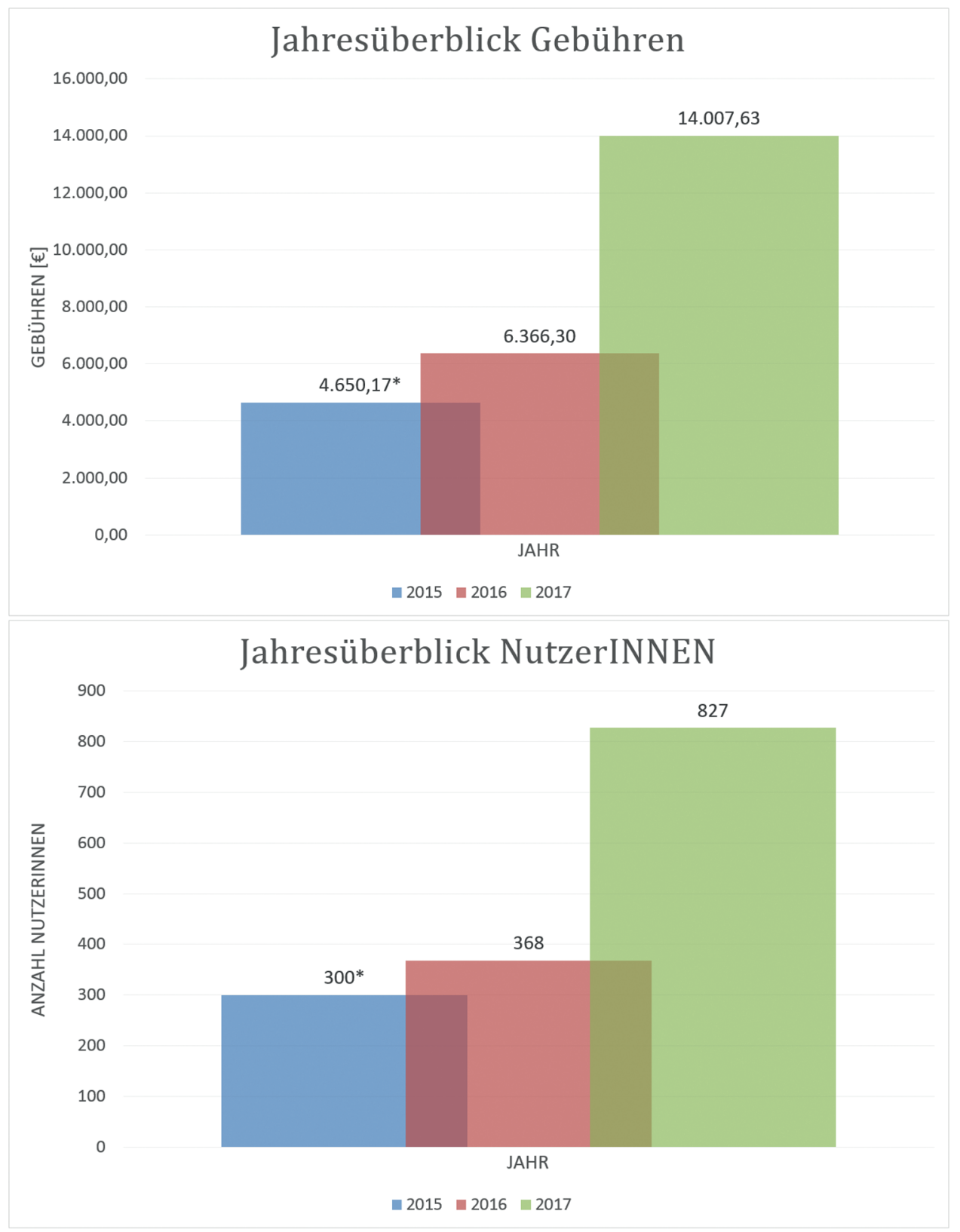

Abb. 5: Entwicklung der Benutzung des SLUB-Makerspace, * = extrapolierte Werte (Stand Januar 2018)

Die Vision des Makerspace in der SLUB Dresden wird nunmehr seit 2015 im Regelbetrieb unterhalten und erfreut sich stark wachsender Beliebtheit bei Nutzern. ${ }^{16}$ Wie die Diagramme in Abb. 5 veranschaulichen, gibt es stark zunehmende Benutzungsgebühren für die einzelnen Geräte, aber auch stark zunehmende Zahlen von Nutzern. Die Erhebung und Abrechnung erfolgt dem oben in Orga-

16 Vgl. Bonte, Lohmeier und Oehm (2014). nisation und Verwaltung beschriebenen Verfahren. Die Gebührenhöhe soll hier lediglich als Richtwert für die Gesamtnutzungsdauer und zur Darstellung der Entwicklung des SLUB Makerspace dienen. Die Absolutwerte der Jahresgebühren sind dabei nur bedingt mit denen anderer Makerspace oder des FabLab zu vergleichen, da sie stark von den jeweiligen Abrechnungssystemen abhängig sind. Die Statistiken für die Jahre 2015 und 2017 sind extrapolierte Werte, welche durch die Aufsummierung der monatlichen Durchschnittsgebühren errechnet wurden. Während 
der Anstieg der Gebühren von 2015 auf 2016 relativ gering ausfällt und hauptsächlich darauf zurückzuführen ist, dass eine vergleichbare Anzahl von Nutzern die gebührenpflichtigen Geräte mehr genutzt hat, steigen die Gebühren von 2016 zu 2017 voraussichtlich sehr stark. Dabei ist zu beobachten, dass die monatlichen Durchschnittsgebühren pro Nutzer in diesem Zeitabschnitt kaum steigen.

Aus diesen Zusammenhängen lässt sich schlussfolgern, dass der Makerspace in den ersten beiden Jahren seines Regelbetriebes zunächst an Nutzungszeit gewonnen hat und im dritten Jahr sehr stark an der Anzahl der ihn nutzenden Personen. Diese Unterscheidung ist wichtig, da beide Phänomene unterschiedliche Entwicklungen beschreiben und jede Entwicklung für sich erstrebenswert ist. Durch den Umstand, dass ein Nutzer längere Zeit im Makerspace verbringt, steigt die Zeit in der er Wissen erlangen oder weitergeben kann; die Bildung von Communities wird so unterstützt. Die steigende Anzahl an Personen verifiziert demgegenüber die steigende Nachfrage und folglich die steigende Attraktivität des Makerspace. Beide Entwicklungen haben natürliche Grenzen und sollten miteinander harmonieren, um negative Auswirkungen auf den Makerspace zu verhindern, beispielsweise die unverhältnismäßig hohe Nutzung einer kleinen elitären Gruppe von Personen. Zusammengefasst sind diese Daten eine Bestätigung der Visionen und Konzepte, die seit dem von Bonte et al. beschriebenen „Experiment Makerspace“ im Jahr $2014^{17}$ entwickelt und umgesetzt wurden.

\subsection{Dauerhafte Wirkung: Nutznießer des SLUB-Makerspace}

Der Makerspace bietet allen registrierten Nutzern der SLUB, den Studenten, den Mitarbeitern der Technischen Universität und darüber hinaus den Bürgern der Stadt und Umgebung, Vorteile. Insbesondere die Professuren der TU Dresden, die den Makerspace für Lehre und Forschung nutzen, profitieren von den Werkzeugen, der neuen Arbeitsumgebung und dem sich hier eröffnenden sozialen Raum. Das sind außer den Ingenieurwissenschaftlern, denen der Makerspace fachlich naheliegt, auch die Geistesund Naturwissenschaftler, denen sich mit dem Makerspace neue Möglichkeiten in der SLUB offenbaren und die dann nicht selten die traditionelleren Angebote der Bibliothek neu zu entdecken beginnen. Themenimpulse im Makerspace führen zu Bildung interdisziplinärer Nutzgruppen, die diese Themen selbständig weiterverfolgen und entwickeln

17 Bonte, Lohmeier und Oehm (2014). und dabei voneinander lernen. So haben sich unter dem Dach der SLUB bislang Experten und Interessierte zusammengefunden, die sich mit 3D-Drucker-Bau, mit 3D-Digitalisierung oder mit 3D-Modellierung auf der Grundlage der Open-Source-Software Blender beschäftigen.

Die Zentralisierung von Ressourcen und Services, die früher über den Campus verstreut waren, führt zu besserer Sicht- und Verfügbarkeit für alle Nutzer. Verschiedene Lehrstühle der TU Dresden stellen dem Makerspace ihre Lehrmaterialien und Maschinen als Leihgaben zur Verfügung, insgesamt wächst das Angebot nicht zuletzt durch substantielle Investitionen der Bibliothek. Auch im Personalbereich ergeben sich aus der Zentralisierung positive Effekte: Durch die personelle Grundversorgung des Makerspace aus der Stammbelegschaft der SLUB und die Widmung von Personalmitteln in Form von Hilfskraftgeldern durch das Fraunhofer IWU konnten die Öffnungs- und Servicezeiten spürbar erweitert werden. Gleichzeitig ist durch die zentrale Verwaltung der Management-Aufwand deutlich gesunken und die Service-Qualität gestiegen.

\subsection{Die Makerspace-Strategie der SLUB ist übertragbar}

Die Existenz des Makerspace ist ein Ergebnis der in der SLUB Dresden stattfindenden strategischen Reflexion der bibliothekarischen Zukunftsgestaltung im Zeitalter der Digitalisierung. Dieses Angebot ist nicht als ein Versuch eines unter zunehmend existentiellen Druck stehenden Dienstleistungssektors misszuverstehen, sich einen neuen Anstrich zu geben. Vielmehr ist der Makerspace auf die ganz konkreten Bedürfnisse einer wissenschaftlich arbeitenden Bibliotheksnutzerschaft ausgerichtet und hat damit Modellcharakter in einer zunehmend digitalen Wissenschaftswelt. Nicht von ungefähr richten inzwischen wissenschaftliche und öffentliche Bibliotheken eigene Makerspaces ein und orientieren sich dabei am Vorbild der SLUB. Als integrales Angebot muss ein Makerspace systematisch ausgebaut werden. Die investiven und personellen Anforderungen sind erheblich und eine entsprechende Entwicklung muss auf der Grundlage guter Planung stehen. Nicht zuletzt dem Wissenszuwachs bei den Mitarbeitern muss besonderes Augenmerk geschenkt werden. In der SLUB haben wir die Weiterbildung der Mitarbeiter im Makerspace in die bibliotheksinterne Aus- und Fortbildung integriert. Persönliche Interessen werden dabei berücksichtigt, denn nur die intrinsische Motivation ist ein Garant für die Befähigung des Teams zum Entwurf und zur Umsetzung exzellenter Dienstleistungs- und Vermittlungskonzepte. Dadurch ist der Makerspace Ort neuartiger Ko- 
operationen der SLUB mit den beschriebenen (vgl. Abschnitt 4.1 Wissenschaftlicher Beirat) Partnern geworden. Es entstanden und entstehen weiterhin nachnutzbare Nutzungs- und Kurskonzepte, Lehrmaterialien und IT-SystemLösungen. Bei Tagungen und Schulungen (HU Berlin, Webinare im Netzwerk Bibliothek des Deutschen Bibliotheksverbands dvb, Bibliothekartag) wurde das erlangte Wissen und die Ressourcen bereits geteilt und weitergegeben.

Da Geräteführerscheine in fast allen offenen Werkstätten durchgeführt werden, sind die als OER veröffentlichen Kursmaterialien für die Übertragung des SLUB-Makerspace-Konzeptes von besonderer Bedeutung. Viele FabLabs oder Makerspaces werden als Verein oder von Freiwilligen betrieben und die Personalressourcen zur Entwicklung aufwändiger Kurse sind dementsprechend knapp. Neben den Nachnutzungsmöglichkeiten haben die Bildungsressourcen jedoch auch einen organisatorischen Mehrwert. Würden andere offene Werkstätten dieselben oder ähnliche Kursmaterialien nutzen, könnte man die Geräteführerscheine gegenseitig anerkennen. Ein Student, der die Universität wechselt, müsste diese dann nicht erneut absolvieren. Dies ist auch im Sinne der Nutzer, da die Geräteführerscheine nicht überall kostenfrei angeboten werden.

\section{Herausforderungen und Ausblick}

Mit steigender Bekanntheit, wachsendem Interesse und sich erhöhenden Nutzungszahlen entstehen neue Herausforderungen für die Bibliothek. Wie im Abschnitt 2.2 Nutzungskonzept beschrieben, wird der Regelbetrieb noch größtenteils manuell und dadurch mit hohem Zeitaufwand von den Beschäftigten des SLUB Makerspace bewältigt. Die zunehmende Anzahl von Anfragen verursacht inzwischen einen mit den zurzeit verfügbaren personellen Ressourcen nicht mehr zu bewältigenden Organisationsaufwand. Hier benötigen wir ein höheres Maß an Automatisation, so dass der eigentlichen Nutzerberatung bzw. -betreuung mehr Zeit gewidmet werden kann. Ähnliches gilt für die Gerätebetreuung. Der Maschinenpark wird kontinuierlich ausgebaut. Um der stetig steigenden Nachfrage gerecht zu werden, aber auch um mit der schnellen Technologieentwicklung Schritt zu halten, wird die Anzahl der FDM-3DDrucker von vier auf insgesamt sieben erhöht. Zusätzlich wird mit zwei Stereolithografie-Druckern auch Hardware für ein neues Druckverfahren angeboten, das neue Möglichkeiten bei der herstellbaren Modellqualität eröffnet und 3D-Druck für neue Nutzergruppen erschließt. Diese Erweiterung des Geräteparks erhöht den Gesamtserviceund -wartungsaufwand für die Geräte. Und schließlich ist auch die Weiterbildung der Beschäftigten im Umgang mit den neuen Technologien mit steigendem Zeitaufwand verbunden, so dass automatisierte Workflows auch hier größere Freiräume schaffen würden.

Unsere Automatisierungskonzepte setzen auf die an der SLUB etablierten bibliothekarischen Workflows und implementieren Software-Lösungen auf und passen sie gemäß den Anforderungen der Makerspace-Benutzung an. So wird momentan an einem Workflow gearbeitet, der die Nutzung des lokalen Bibliothekssystems LIBERO zur Verwaltung der Arbeitsschutzbelehrungen und Geräteführerscheine für jeden einzelnen Nutzer ermöglicht. LIBERO bietet seit der kürzlich in der SLUB eingeführten Version 6 eine API, die genutzt werden kann, um beliebige Daten aus der Datenbank abzufragen oder in sie hineinzuschreiben. Die Programmierung entsprechender Web-Applikationen ist damit grundsätzlich möglich (und nur noch ein Ressourcen-, kein technisches Problem mehr). Die Realisierung dieses Workflows ist Voraussetzung für ein visionäreres Ziel: die Identifikation einzelner Nutzer mit ihren SLUBBenutzerkarten direkt am gebuchten Gerät, das, abhängig von Bedingungen (Ist die Arbeitsschutzbelehrung noch gültig? Ist der Geräteführerschein absolviert worden? Ist das Konto auch nicht anderweitig - beispielsweise durch ausstehende Gebühren - gesperrt?), für die Benutzung freigegeben bzw. samt seinem dazugehörigen PC gestartet wird.

Ohne solche Entwicklungen ist der Wechsel von einem „WDIFY (We Do It for You)“ hin zu einem „DIY (Do It Yourself)“ nur sehr schwer realisierbar. Um den Wandel hin zu Digitalisierung in Gesellschaft und Wissenschaft zu befördern und mitzugestalten, müssen die wissenschaftlichen bzw. wissenschaftsunterstützenden Einrichtungen selbst digital werden, auch auf den fundamentalen Infrastruktur- und Organisationsebenen.

Die grundsätzliche Öffnung der Bibliothek gegenüber der Digitalisierung und den digitalen Wissenschaften bzw. die tiefere Reflexion der Bedeutung einer solchen Öffnung lassen Konzepte wie das des Makerspace in einem anderen Licht erscheinen. Wurde eingangs festgestellt, dass Bibliotheken Orte des Fertigens, Erzeugens, des „Machens“ waren, so ist in unseren Ausführungen deutlich geworden, dass der Makerspace in der SLUB Dresden zunächst einmal als eine von den übrigen bibliothekarischen Angeboten relativ klar abgegrenzte Infra- und Servicestruktur entwickelt worden ist. Da unser Konzept Makerspace in seiner Entstehung vom Begriff der Bibliothek hergeleitet wurde, ist es gerechtfertigt, von einer „Bibliothek in der Bibliothek" zu sprechen. ${ }^{18}$ Unserer Ansicht nach lässt sich dieser

18 Vgl. hierzu Bonte (2016) 90. 
Begriff des Makerspace jedoch zurückübertragen auf die gesamte Bibliothek, so dass wir Bibliothek künftig als einen großen Makerspace verstehen. Das entspricht grundsätzlich unseren Überlegungen, dass Bibliotheken sich vom engen Verständnis als Einrichtungen der Informationsrecherche und Literaturversorgung weiterentwickeln müssen und dabei den gesamten Forschungszyklus in den Blick nehmen müssen. ${ }^{19}$ Die Dienstleistungen, die wir in diesem Zusammenhang anbieten bzw. vermitteln, müssen sich auf alle Phasen dieses Zyklus beziehen. ${ }^{20}$

Gemäß diesem Verständnis entwickeln wir in der SLUB den Makerspace „in die traditionelle Bibliothek hinein“. Über Prototyping hinaus - und unter Einbeziehung von Kollegen, die nicht zum Kernteam Makerspace gehören - erweitern wir den Makerspace derzeit um die neue Werkstatt M3 für audiovisuelle Technik in der Tonund Bildproduktion. Ziel ist es, Werkzeuge, Arbeitsmittel und Methoden der Bibliothek, die bisher hinter den Kulissen genutzt werden (z. B. die Mediathek), auch Nutzern für eigene Projekte zugänglich zu machen. Das Netzwerk Schreiben, das bereits im Zusammenhang mit der Smart ${ }^{3}$ Academy erwähnt wurde, soll in Zukunft ebenfalls als Makerspace-Bereich firmieren. Hier schließt sich gewissermaßen der Kreis, den wir mit der Lösung von der ausschließlich textuellen Wissensvermittlung begonnen haben $\mathrm{zu}$ beschreiten: Wir sind wieder beim Textuellen angelangt, sehen es aber nun im Zusammenhang mit allen anderen fundamentalen technologischen, medialen, sozialen und organisatorischen Fähig- und Fertigkeiten, an deren Bildung und Förderung wir Bibliotheken uns gerne beteiligen.

\section{Literaturverzeichnis}

Bonte, Achim (2016): Vorstoß in neue Wissensräume - Makerspaces im Leistungsangebot wissenschaftlicher Bibliotheken. In: Strategien für die Bibliothek als Ort: Festschrift für Petra Hauke zum 70. Geburtstag, hg. v. Andrea Kaufmann, Klaus Ulrich Werner und Konrad Umlauf. Berlin, Boston: De Gruyter Saur.

Bonte, Achim; Lohmeier, Felix; Oehm, Lukas (2014): Experiment Makerspace in der SLUB. In: BIS - Das Magazin der Bibliotheken in Sachsen, 7(2), 74-76. Verfügbar unter http://nbn-resolving.d e/urn:nbn:de:bsz:14-qucosa-148293.

DBV (2014): Best-Practice-Wettbewerb 2014: Thema: Vermittlung von Informationskompetenz an fortgeschrittene Studierende, Promovierende, Wissenschaftlerinnen und Wissenschaftler. Deutscher Bibliotheksverband. Verfügbar unter http://www.bib liotheksverband.de/fachgruppen/kommissionen/informations

19 Vgl. Lohmeier, Mittelbach und Stöhr (2016).

20 Vgl. hierzu unser Library Services Mindmap, Mittelbach (2016). kompetenz/best-practice-wettbewerb/2014.html, zugegriffen am 30.09.2017.

Dresden Technologieportal (2017): Geräte - Makerspace - SLUB Dresden (SLUB Makerspace) Dresden Technologieportal. Verfügbar unter https://tp.dresden-concept.de/de/equipment/ byunit/id/1774/1/100, zugegriffen am 30.09.2017.

Gartner, Johannes (2012): MakerBot: Metallobjekte mittels Gussform erzeugen. In: 3Druck.com - Das Magazin für 3D-Drucktechnologien.

GoAnimate (2017): GoAnimate. Verfügbar unter https://goanimate.co $\mathrm{m} /$, zugegriffen am 30.09.2017.

Hochschulrektorenkonferenz (Hrsg.) (2013): Hochschule im digitalen Zeitalter: Informationskompetenz neu begreifen - Prozesse anders steuern. Entschließung der 13. Mitgliederversammlung der HRK am 20. November 2012 in Göttingen. Bonn (Beiträge zur Hochschulpolitik).

Kaiser, Melanie (2016): Von der Idee zur Umsetzung - so wird's gemacht: 3D-Kunststoffdruck. Dresden.

Kaiser, Melanie; Fröhner, Uta (2016): Von der Idee zur Umsetzung so wird`s gemacht: Lasercutter. Dresden.

Krzywinski, Jens (2014): Wissens.Werkstatt „Printed Phenomena“ und „Folded Spaces“. Sommerschule des Dresden Design Hub der TU Dresden experimentierte im Makerspace der SLUB. In: BIS - Das Magazin der Bibliotheken in Sachsen, 7 (2), 77-78. Verfügbar unter http://nbn-resolving.de/urn:nbn:de:bsz:14-qu cosa-148302.

Lohmeier, Felix; Mittelbach, Jens; Stöhr, Matti (2016): Informationsservices auf Augenhöhe - So können Bibliotheken den Forschungsprozess proaktiv unterstützen. In: Handbuch Informationskompetenz. 2. Aufl., hg. v. Wilfried Sühl-Strohmenger und Martina Straub. Berlin, Boston: De Gruyter Saur (De Gruyter Reference).

Lynda.com (2017): Lynda.com. Verfügbar unter http://www.Lynda.co m, zugegriffen am 30.09.2017.

Mittelbach, Jens (2016): Library Services: A concept of information services in academic liraries aligned to the research cycle.

Mittelbach, Jens; Muschalek, Antonie (2015): Wissensbar: Experten beraten passgenau. In: BuB: Forum Bibliothek und Information, 67(6), 374-76. Verfügbar unter https://www.researchgate.net/ publication/280620882.

Morrison, Campbell; Beranek, John (2017): MRBS: Meeting Room Booking System.

Noenning, Jörg Rainer; Oehm, Lukas; Wiesenhütter, Sebastian (2014): Fablabs für die Forschung, 33-47. Dresden.

Petzold, Judith (2016): Stadtbibliothek Köln - Bibliothek des Jahres 2015. In: BIBLIOTHEK - Forschung und Praxis, 40(2), 28393. doi: 10.1515/bfp-2016-0035.

Schäfer, Anna Maria (2012): Das Inverted Classroom Model. In: Das inverted classroom model: Begleitband zur ersten deutschen ICM-Konferenz, hg. v. Jürgen Handke und Alexander Sperl, 3-12. München: Oldenbourg.

Schuldt, Karsten (2017): Makerspace oder nicht - eine Frage der Community. doi: 10.18452/2347.

SLUB Dresden (2017a): Gerätebuchung. Verfügbar unter https:// www.slub-dresden.de/service/arbeitsplaetze-arbeitsraeume/sl ub-makerspace/geraetebuchung/, zugegriffen am 30.09.2017.

SLUB Dresden (2017b): Netzwerk Schreiben. Verfügbar unter https:// www.slub-dresden.de/service/netzwerk-schreiben/, zugegriffen am 30.09.2017. 
SLUB Dresden (2017c): SLUB Raumbuchungssystem. Verfügbar unter https://raumbuchung.slub-dresden.de/week.php?area=10, zugegriffen am 30.09.2017.

Zarre, Doris (2017): Volkswagen Autoeuropa setzt auf 3D-Druck bei Werkzeugherstellung für Montage. In: 3Druck.com - Das Magazin für 3D-Drucktechnologien.

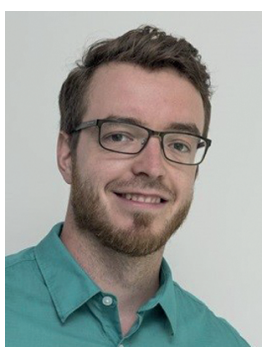

\section{Jonas Tiepmar}

Sächsische Landesbibliothek - Staats- und Universitätsbibliothek Dresden (SLUB)

D-01054 Dresden

jonas.tiepmar@slub-dresden.de

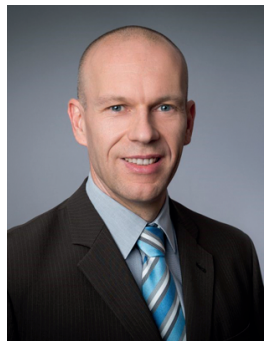

\section{Jens Mittelbach}

Sächsische Landesbibliothek - Staats- und Universitätsbibliothek Dresden (SLUB)

D-01054 Dresden

jens.mittelbach@slub-dresden.de

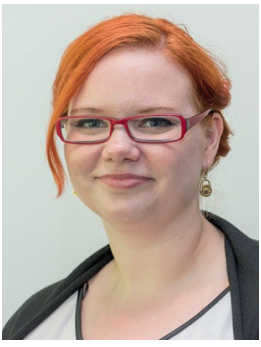

\section{Melanie Kaiser}

Sächsische Landesbibliothek - Staats- und Universitätsbibliothek Dresden (SLUB)

D-01054 Dresden

melanie.kaiser@slub-dresden.de

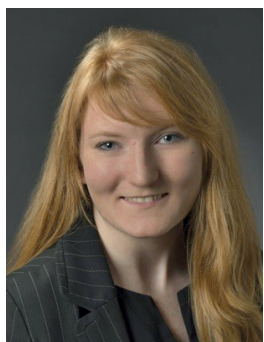

\section{Daniela Dobeleit}

Sächsische Landesbibliothek - Staats- und Universitätsbibliothek Dresden (SLUB)

D-01054 Dresden

daniela.dobeleit@slub-dresden.de

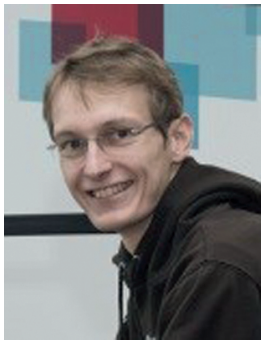

Paul Schwanse

Sächsische Landesbibliothek - Staats- und Universitätsbibliothek Dresden (SLUB) D-01054 Dresden paul.schwanse@slub-dresden.de

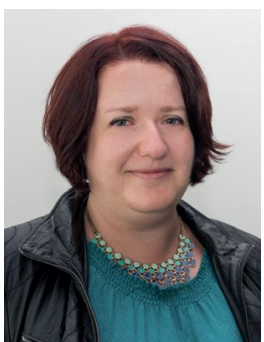

\section{Uta Fröhner}

Sächsische Landesbibliothek - Staats- und Universitätsbibliothek Dresden (SLUB) D-01054 Dresden uta.froehner@slub-dresden.de

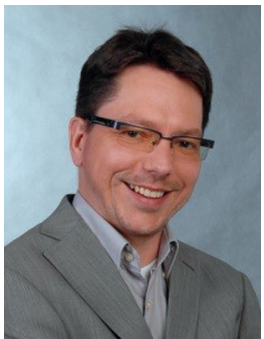

\section{Maik Jähne}

Sächsische Landesbibliothek - Staats- und Universitätsbibliothek Dresden (SLUB) D-01054 Dresden maik.jaehne@slub-dresden.de 